Research Article

\title{
Flexible-Robotic Reflector for Aerospace Applications
}

\author{
Nir Shvalb, Boaz Ben-Moshe, Oded Medina, and Raz Itzhaki Tamir
}

Aerospace and Nano-Satellite Research Center, KఓCG Lab., Ariel University, Israel

Correspondence should be addressed to Boaz Ben-Moshe; benmo@g.ariel.ac.il

Received 6 August 2015; Accepted 17 November 2015

Academic Editor: Shih Yuan Chen

Copyright (C) 2015 Nir Shvalb et al. This is an open access article distributed under the Creative Commons Attribution License, which permits unrestricted use, distribution, and reproduction in any medium, provided the original work is properly cited.

Existing dish based antennas tend to have geometric morphologic distortion in the surface due to drastic thermal changes common in the space environment. In this paper we present a new concept for a dynamic antenna specially designed for communication satellites. The suggested flexible-robotic antenna is based on a dual-reflector structure, where the subreflector has a complex surface shaping robotic mechanism allowing it to fix most of the morphologic errors in the main reflector. We have implemented a set of searching algorithms allowing the hyper redundant robotic subreflector to adapt its surface to the morphologic distortions in the main reflector. The suggested new antenna was constructed and tested in an RF room in which it was able to fix the loss caused by distortion in the main reflector to the original gain in less than an hour.

\section{Introduction}

Satellite communication requires an ever growing bandwidth. Uplink and downlink demands to satellites are increasing as higher video resolution becomes available in a growing number of devices and applications. An extensive literature is abundant on improving satellite communication technology. This paper issues the problem of designing a flexible antenna which enables an improved link budget with smaller and lighter form factor. The term flexible antennas will refer to antennas that can adjust to a required shape and several times during their operational life. The need for self-adjustable antennas arises in environments where manual adjustments are impossible (e.g., space). Launching a satellite is conceptually performed by applying a controlled explosion. During launch, which takes just a few minutes in a low Earth orbit and up to several weeks in a geostationary orbit the satellite is exposed to vibrations and shocks that can damage its structure. In order to avoid damage a supporting structure is required which increases the satellite total weight and size. Thus, conventional satellite launch is a significant portion of the total cost of a communication satellite program.

Expandable antennas solve this problem by holding the antenna in a folded configuration during launch and deploying it when the designated location is reached. Thus, the supporting structure is not required anymore. Yet the resulting shape of an expandable antenna is never as accurate as the shape of a rigid antenna. This may cause a considerable effect on performance (e.g., the antenna gain). A flexible subreflector can regain the losses (gaining the gain) by fixing the wavefront. Yet analytic methods are applicable mainly for simple and symmetric shapes. On the other hand an exhaustive checking of all possible combinations is only practical for problems with a small number of degrees of freedom, due to high exponential cardinality of the configuration space (with respect to the number of degrees of freedom). Evolutionary algorithms may be applied to solve more complex problems. Generally speaking, evolutionary antenna design is carried out stating with a simple antenna design. Adding or changing elements in a semirandom manner should result in several new shapes of antenna. These are then evaluated to determine how they meet with the design requirements (e.g., antenna pattern requirements and total gain requirements). Accordingly, a numerical score is calculated for each. Then, in a similar way to natural selection, some antenna candidates with the worst scores are discarded resulting in a smaller population of designs having the highest score. The algorithm repeats the process to create a more successful antennas population until a termination criterion is met. 
In this paper an innovative new design for adaptive dualreflector antenna is presented. To the best of our knowledge, this is the first work which presents a many-degree-offreedom robotic subreflector with an efficient heuristic for searching the huge configuration space for an optimal surface configuration. This design allows overcoming morphological distortions in the main reflector and adaptive beam-shaping which may improve the overall performance and robustness of communication satellites.

The rest of the paper is organized as follows: after Section 1 a short survey of related works is given. Then, the general structure and mechanism of the flexible antenna are presented. Then, we elaborate on the heuristics used for controlling and optimizing the robotic antenna. Finally, a set of experimental results are given followed by a short conclusion discussing the possible applications and future work regarding the robotic space antennas.

\section{Related Works}

Designing better and more robust antennas for communication satellites is a research challenge which occupies many researchers; see [1-3] for updated surveys regarding space grade antennas.

The process of designing an optimal antenna is still somewhat fuzzy and might require the use of artificial intelligence tools. In [4], for example, the researchers obtained a desired side-lobe for an aperiodic antenna array. Particularly, the researchers find a thinned designed array that produces the lowest maximum relative side-lobe level. The first fully evolved antenna design appeared in [5], where the researchers introduce an efficient monopole wire antenna for aerospace applications. The antenna had four identical arms, with each arm rotated $90^{\circ}$ from its neighbors. To produce this type of antenna, the algorithm computes a single arm shape at a time these are evaluated by building a complete antenna using four copies of the evolved arm.

Robotic antennas with a limited number of degrees of freedom may allow the use of brute-force methods for searching the whole configuration space, yet exhaustive checking of all possible combinations is only practical for problems with a small number of degrees of freedom, such as small antenna array design (see, e.g., [6]).

Our main goal here is to design a many-degree-of-freedom surface robot that will serve as a subreflector antenna. In the course of planning the antenna's mechanical design we considered several conceptual options [7] with regard to their backbone structure: (i) hyper redundant rigid robots: which are built in a traditional rigid link and joint concatenation manner but typically have full dexterity in almost all configurations like the robotic worm introduced in [8]; (ii) soft robots: which are mechanisms that possess a compressible flexible backbone (examples are scarce but see [9-11]); (iii) flexible robots: that possess a noncompressible flexible backbone such as the robotic surface introduced in [7] or the physical interaction board introduced by Vink et al. in [12]. In this paper we shall discuss a flexible-robot antenna which conceptually is designed as Vink's pin-board [12].
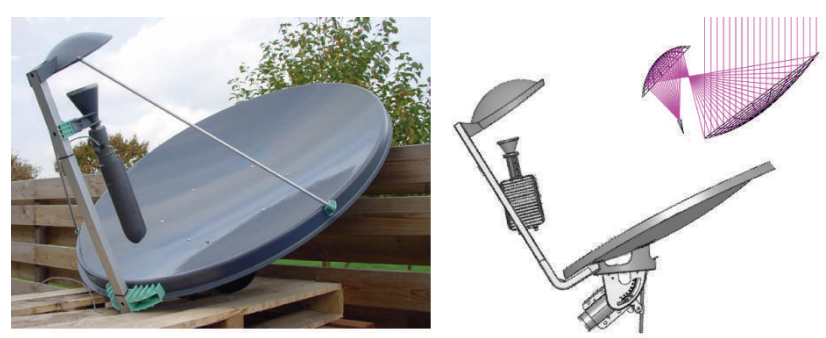

FIgURE 1: The general structure of a dual-reflector antenna. The static subreflector (shown on the upper part of the figure) was replaced with a dynamic surface mechanism.

\section{The Flexible-Robotic Subreflector}

In this section we present the main structure of the adaptive satellite antenna which is based on a robotic subreflector. The general structure of a fix (i.e., static) dual-reflector antenna is shown in Figure 1, while dual-reflector antenna with a robotic subreflector is shown in Figure 5.

In order to design a robotic subreflector we needed to overcome two major challenges:

(i) How to construct an RF flexible surface.

(ii) How to design a robotic system which has the needed degrees of freedom (DoF) in order to shape the RF reflector surface to all the possible configurations. We started by solving each problem separately.

3.1. Flexible RF Subreflector. There are many types of RFreflecting materials, yet in general they are all originally designed to be applied on rigid structures and most of them are not suitable for flexible surfaces. For example, consider an aluminum foil; although it is flexible and easy to shape it can not be applied on a dynamic reflector, as the changes in the surface of the reflector will cause the foil to crumple, bend, and even break. In general we need a silicon-nature surface which is both flexible and smooth. In order to fabricate such subreflector we first ignored the RF-reflection requirement. We tested a wide range of $R T V$ or silicon-like materials. We then tested the dynamic nature of those surfaces using a simple robotic subreflector mechanism shown in Figure 4. Given an adequate design for a flexible surface we have started the process of converting it to be RF-reflecting. Our first approach was to apply an RF-reflecting paint and several types of RF-reflecting foils on the flexible surface as shown in Figure 2, yet this approach was insufficient as the paint tends to crack and break from the surface as the reflector flexes.

An alternative method was to fabricate a single thin layer of silicon-like membrane (shaped like the original subreflector). We have used an elastic netlike fabric and perform a process of aluminum evaporation over it, allowing the fabric to have a high RF-reflecting property. Finally, the fabric-net was attached to the membrane and another layer of siliconlike membrane was formed on it, locking the RF fabric-net between the silicon-like membranes. The net structure of the fabric allowed the two membranes to be glowed through 

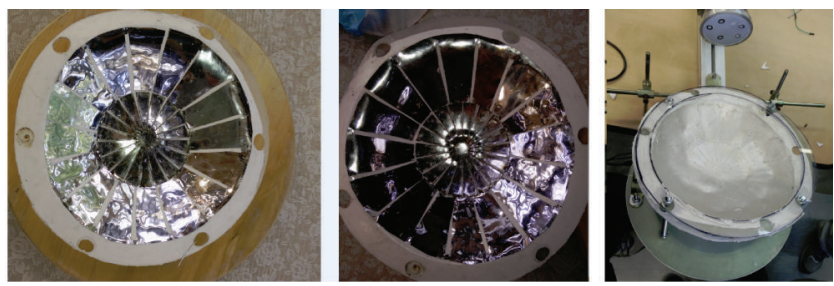

FIGURE 2: Several RF-reflecting materials were tested; they all had high reflecting results, but connecting them to the flexible surface was troublesome.

the net-holes without reducing the RF-reflection property of the fabric. The general structure of the advanced version of the robotic subreflector is shown in Figures 4 and 6.

3.2. Multiactuators Subreflector. We designed and fabricated a subreflector with 24 degrees of freedom (DoF). These are used to reshape the subreflector curvature. The linear motors were oriented perpendicularly to the back plane, to which they were attached. The actuators were located homogeneously on the plane constraint to the existence of 14 peripheral actuators that are vital in order to enable the antenna's edges to change their shape. These were estimated to have a large effect on the resulting total antenna gain due to RF diffraction phenomenon.

Theoretically, in order to enable full dexterity each actuator should be given 5 passive degrees of freedom, those of the joints connecting it to its surroundings. This is required in order to prevent the motors from exerting torsional torque on the dynamic surface.

In the first model the base of the motors was fixed to the back plane $(0 \mathrm{DoF})$, while the shaft was attached via a short silicon ribbon enabling full orientation change ( $3 \mathrm{DoF}$ ) and a minimal translation (since the ribbon was only $1 \mathrm{~mm}$ long this amounts to an additional $2 \mathrm{DoF}$ ). In the second model the base of the motors was attached to the back plane via U-joints (2 DoF) while their distal ends were attached to the surface via spherical joints (3 DoF).

We used Pico linear servo for the first model (see Figure 3) and Firgelli linear servo motors for the second, Figure 4. Both are standard RC servos with resolution of $\sim 0.5 \mathrm{~mm}$. These were connected to a 32-servo controller actuated by a PC using MATLAB.

\section{Subreflector Optimization}

In this section we present the general search heuristic for finding the optimal configuration for the subreflector. More formally, we would like to find the configuration vector $(V)$ for each of the $n$ actuators, such that $V$ maximizes the overall gain of the antenna, as computed by the feedback channel of the antenna. Each actuator has an integer range $[0, K]$ mapped to a linear actuator of $T \mathrm{~mm}$, with accuracy of $T / K \mathrm{~mm}$. The cardinality of the configuration space is $O\left(K^{n}\right)$. In the constructed antenna each of the 24 actuators had a dynamic range of 1500 values (linear servo motor) and a range of $30 \mathrm{~mm}$ movement, allowing an accuracy of 20 microns per

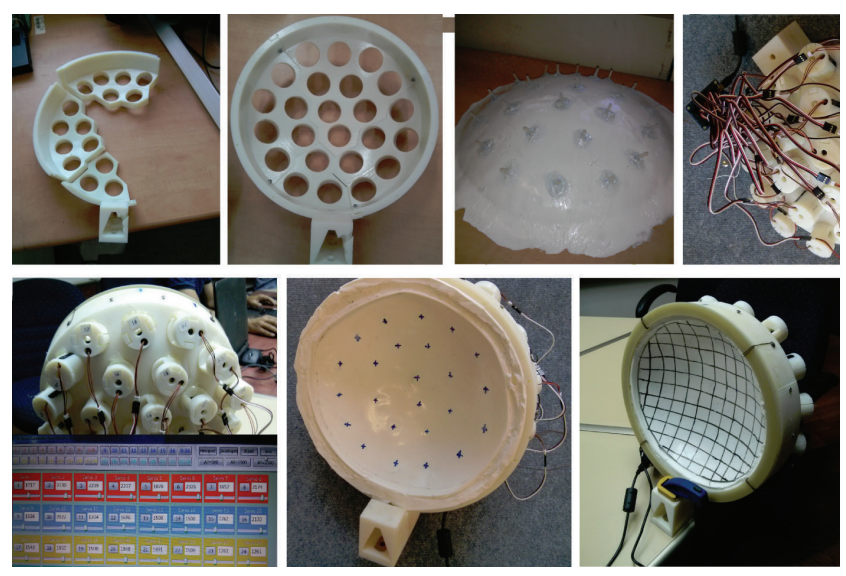

FIGURE 3: The early printed model of the dynamic subreflector. It was mostly printed using a 3D-printer. Each position accommodated a round shape housing which simplified the assembly process.
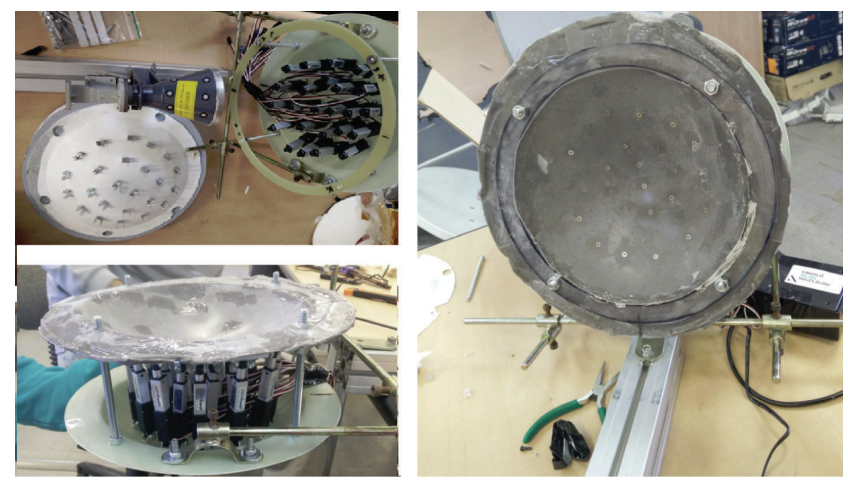

FIGURE 4: The advanced model of the dynamic subreflector. It was mostly made of complex materials, and each linear actuator was placed and locked to the surface allowing a tight structure while maintaining the flexibility of the dynamic RF membrane.

actuator, presenting a theoretical configuration space of size $1500^{24}>10^{75}$ which is close to the number of atoms in the observable universe (considered to be $10^{80}$ ).

It is essential to exclude invalid configurations (e.g., configurations which might damage the subreflector). The resulting space is referred to in literature as the free configuration space or valid configurations. We define such a valid configuration using the following two rules:

(i) For each pair of adjacent actuators (i.e., neighbors) the slope is restricted to a given range.

(ii) Consider the set of facets spanned by triplets of adjacent actuators. The angle between adjacent facets is restricted to a given range (i.e., not too sharp).

A configuration $V$ is considered valid if and only if it satisfies both rules. While the first rule is simple to test, the second rule is somewhat hard to implement; we have used a relaxed version of this rule using a Delaunay triangulation [13] over the $z$-value of the actuators and test its first- and second-degree slopes with respect to the maximal allowed range. Below is a simple heuristic. 


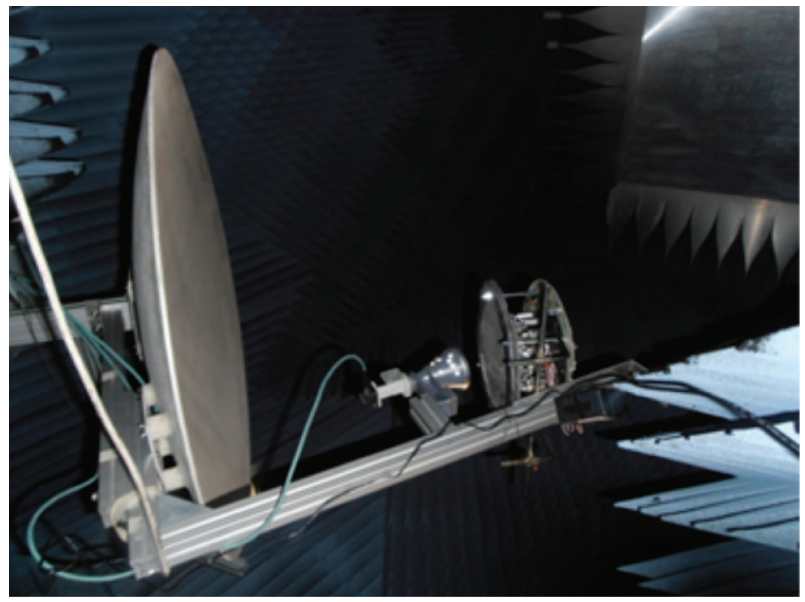

(a)

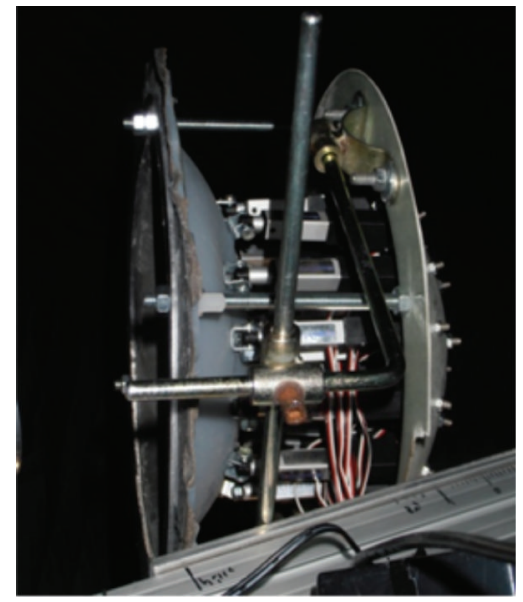

(b)

FIgURE 5: The dual-reflector antenna with the dynamic subreflector. (a) The overall system during an RF test. (b) The flexible subreflector.

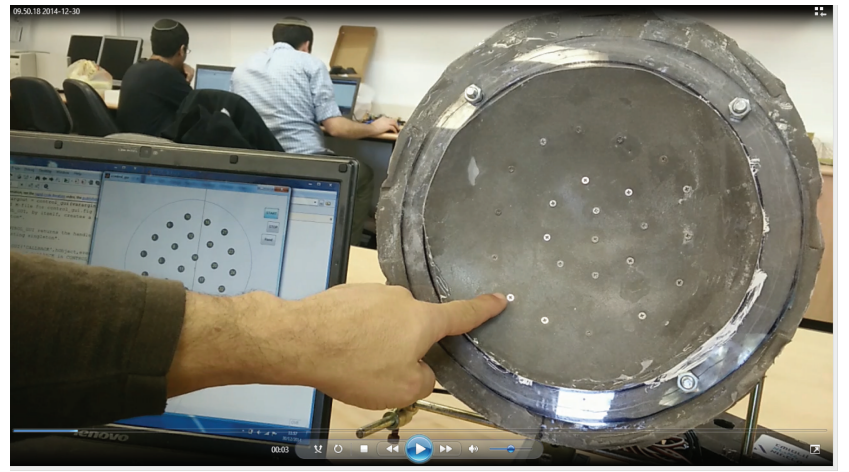

FIGURE 6: The final model of the adaptive subreflector, with its controlling software.

4.1. Naive Search Heuristic. We start by presenting a simple greedy-randomized heuristic (Algorithm 1) for searching the configuration space in order to find a maximal configuration vector, where $f(V)$ is the objective function, estimating the benefit of the configuration point $V, \epsilon$ is some positive epsilon value, and $V_{\text {init }}$ is the starting configuration vector, usually the configuration of the original subreflector. In order to welldefine the above heuristic we need to define the following functions.

(i) Graduate Decent (V). It performs a greedy search starting from $V$ and looking for a local improvement at each actuator. The order of the actuators on which the greedy search is being performed is important and is being set randomly each time.

(ii) Random Valid Configuration Vector. This process has two stages. (i) Let $R$ be a random order of the actuators. (ii) For each $r \in R$ randomly pick a valid value according to the values already chosen.
Result: Optimal Configuration Vector for the sub-reflector

(1) initialization Let $A N S=V_{\text {init }}, C U R R=A N S$;

(2) while $(f(O P T)-f(A N S)>\epsilon)$ do

(3) Perform graduate decent for CURR;

(4) if $(f(C U R R)>f(A N S))$ then

(5) $\quad A N S=C U R R$;

(6) end

(7) Let CURR be a random valid configuration vector;

(8) end

(9) return ANS

Algorithm 1: A naive algorithm for searching a maximal configuration vector.

4.2. Advance Search Heuristic. The naive heuristic (Algorithm 1) presented above may have a runtime which is too long; this is mainly due to two elements:

(i) Stateless. The naive method is not storing good configuration vectors (which have already been found).

(ii) Blind Gradient Decent. It is performed serially and therefore both slow and insufficient in terms of undersampling the configuration space.

We now present the improved method (Algorithm 2) for searching the optimal configuration of the subreflector which overcomes the above drawbacks.

In order to define the above heuristic the following functions and attributes should be well-defined.

(i) Random Maneuver $(V, U)$. This function computes a monotone path from configuration vertex $V$ to configuration vertex $U$ where the rate of change for every actuator is computed randomly. (In most cases we have tested the range of the rate of change which was fixed to $[10 \%, 100 \%]$.) 


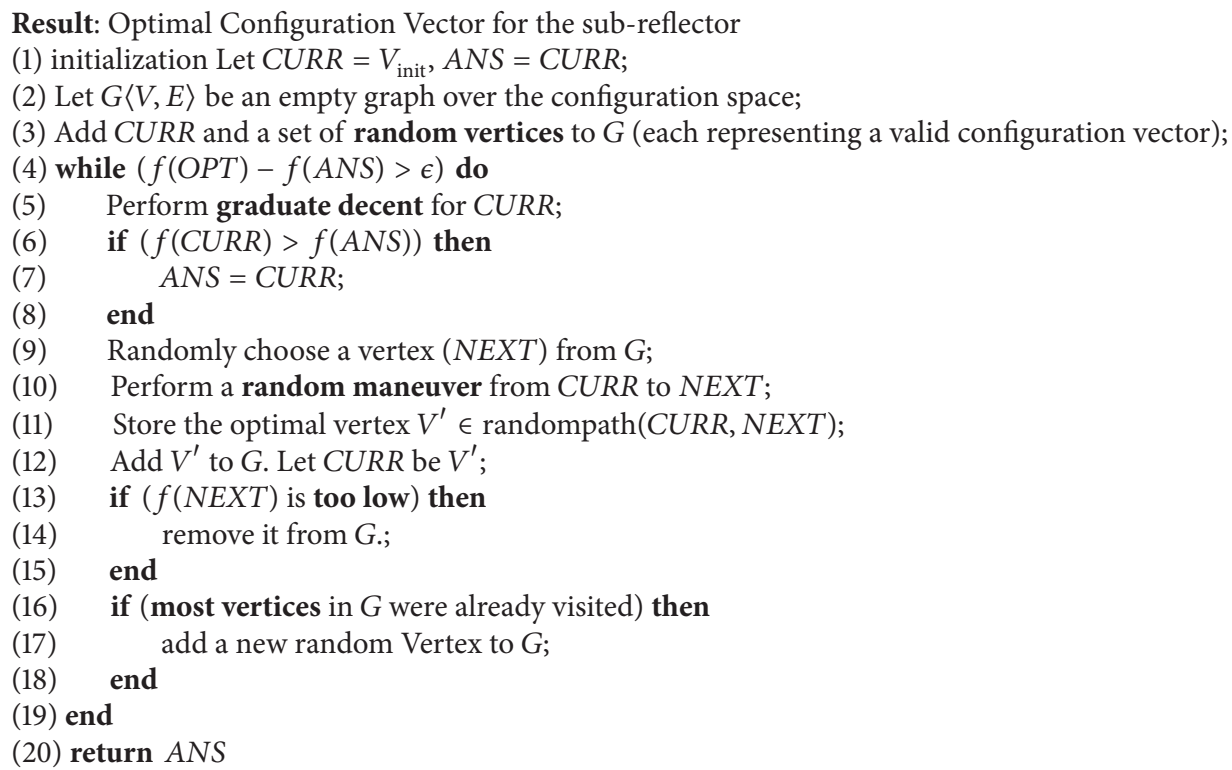

Algorithm 2: A generic algorithm for searching a maximal configuration vector.

(ii) Random Vertices. A starting size of $G$ of the actual size is not that important; any value of $[50,1000]$ should be fine. This set may also include some predefined configuration vectors, which are known as good starting points.

(iii) Too Low. It can be seen as a value which is lower than [50\%, 90\%] of the vertices in $G$.

(iv) Most Vertices. Values in the range of [60\%, 90\%] seem to work fine.

Both versions of the algorithms were implemented in MATLAB. In real scenarios the algorithm computational complexity is redundant as moving the actuators requires significantly more time than computing the algorithm step. Therefore the algorithm runtime depends on the number of serial changes performed in the actuators position. Each change in the actuators positions requires about $1-8$ seconds on average. The advanced version of the algorithm was able to converge the optimal configuration in less than 1 hour (less than 1000 steps).

\section{Experimental Results}

In order to estimate the suggested algorithms we have performed a set of experiments. We started with fabricating a distorted main reflector and performed a $3 \mathrm{D}$ laser scan for both the original and the distorted reflectors; the errors in the distorted reflector are shown in Figure 8.

5.1. Simulation Experiments. We have used the TICRA [14] $\mathrm{RF}$ simulator in order to model both the original and the distorted antennas. This RF simulator has a near-field simulation package and is commonly used for designing and evaluating complex satellite antennas. We have then computed and fabricated an optimal (fixed) subreflector for the distorted reflector. Using TICRA [14] simulator we were able to show that the advance algorithm is significantly faster than the naive one and can search the configurations space efficiently. Yet the RF simulation results using TICRA were not fully aligned with the RF room results; in particular, the optimal (fixed) subreflector which was specially designed for the distorted subreflector performed poorly in the real RF experiments.

5.2. Experiment Setting. In order to test the actual performance of the suggested dynamic robotic antenna and evaluate the search algorithms, we have used a certified RF room for MTI Wireless Edge [15]. The transmission frequency was set to $14.5 \mathrm{Ghz}$ (the center of $k u$-band frequency range). Along with the original 1.2-meter main reflector a distorted main reflector was constructed with errors as seen in Figure 8. The errors were computed as a sine function with errors which are about $1 / 3$ the wavelength. The actual experiment setting is shown in Figure 7. The fine changes in the subreflector are shown in Figure 10; the changes in the subreflector height (from the original position of the subreflector) are presented as a topographic map. This map was computed using a smoothing process over the 24 actuators, representing the subreflector flexible surface (membrane).

5.3. Algorithm Results. The main results of the experiments in the RF room are shown in Figure 9. One can see that distorted subreflector caused a gain loss of about $5 \mathrm{~dB}$ and the dynamic subreflector was able to gain back the original antenna gain in the frequency range in which it was optimized over. In fact, in the case shown in Figure 9, the dynamic subreflector was 


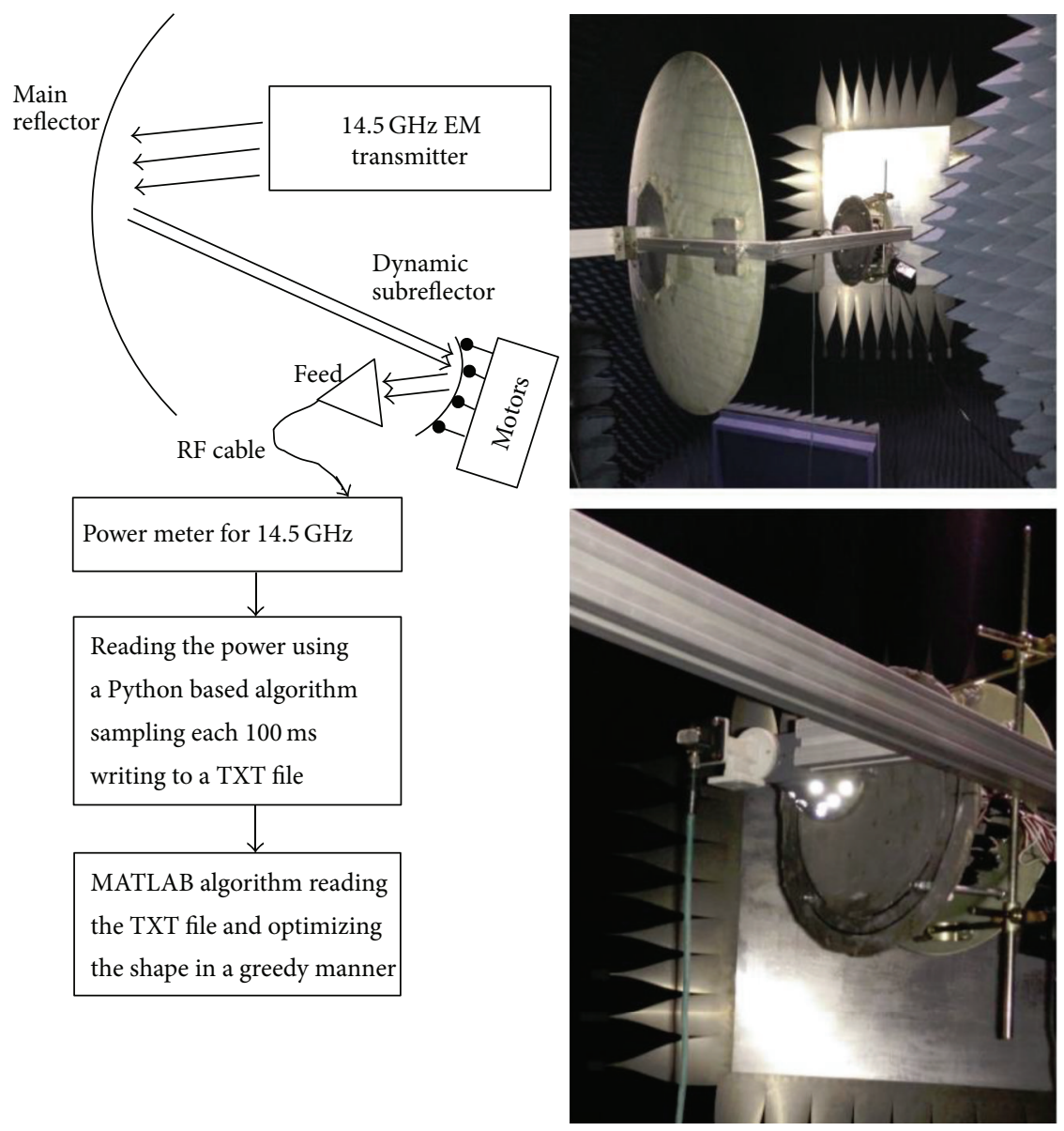

FIGURE 7: The general setting of the search algorithm as was implemented in the RF room experiment.

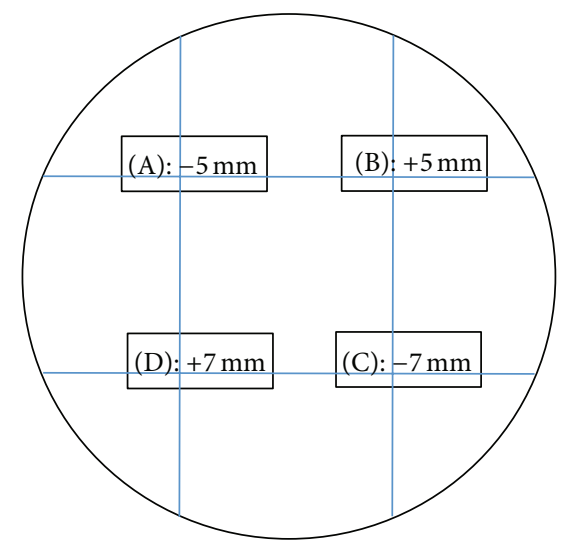

(a)

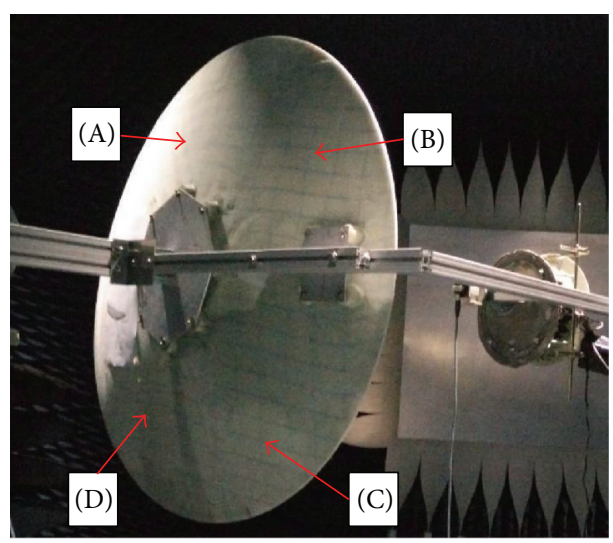

(b)

FIGURE 8: The general setting of the experiment. (a) The distorted main reflector with the errors. (b) The distorted main reflector with the dynamic subreflector during the experiment in the RF room. 


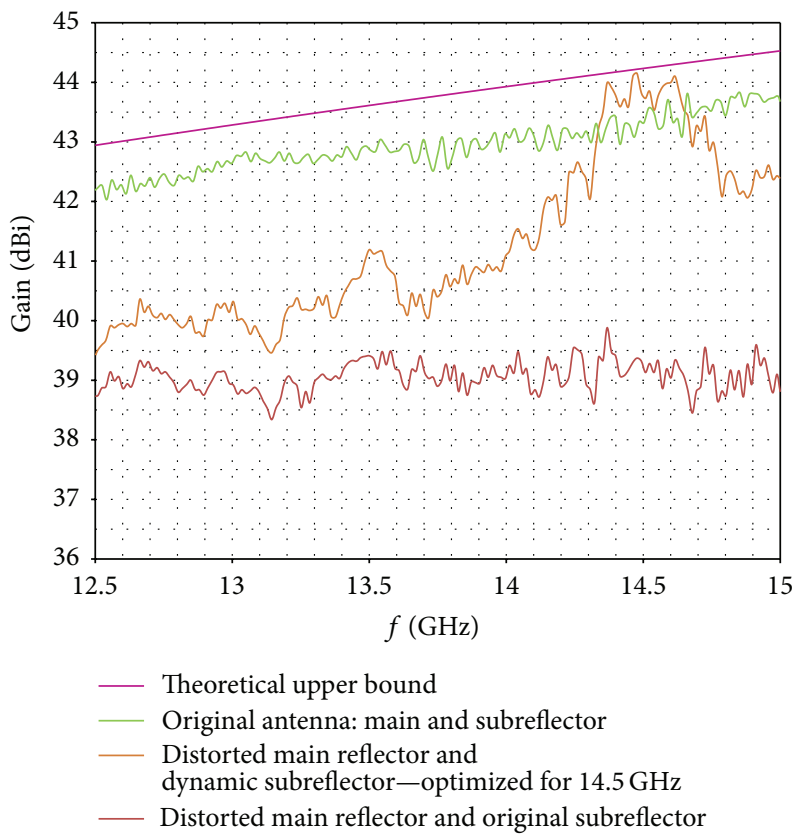

FIGURE 9: The main results of the dynamic subreflector. The dynamic subreflector was able to improve the overall antenna gain in about $5 \mathrm{~dB}$ which is shown at $14.5 \mathrm{Ghz}$; this even improves the original antenna performance in about $1 \mathrm{~dB}$ gain. The improvement is valid for about $\omega=330 \mathrm{Mhz}$ frequency-band, ranging from 14.33 to 14.66 Ghz.

able to improve the original setting in almost $1 \mathrm{~dB}$ and reached very close to the theoretical upper bound of the possible antenna gain. We have also tried to optimize the subreflector manually; this process has led us to suboptimal results each time.

\section{Conclusion}

In this paper we present a new concept of an adaptive aerospace antenna, which can overcome distortions in the main reflector. The presented algorithm allowed us to improve the link budget in 3-7 dB and as shown in Figure 9 in some cases the adaptive subreflector has gained better results than the original antenna. The overall process of finetuning the robotic subreflector required less than an hour. This may seem like a time-consuming task yet tuning a satellite after launching (or during maintenance procedure) is a process which normally requires hours; therefore, the process of optimizing the robotic subreflector in an hour is applicable for many real-life satellite applications. The ability to adapt the subreflector opens a wide range of applications including beam-shaping, frequency optimization, and null steering. But most important it may allow using smaller and lighter main reflectors which before were simply not suitable for aerospace applications due to possible distortions. Such antennas may be significantly cost-effective to many satellite communication applications. For future plans we would like to construct a space-qualified antenna system with a flexiblerobotic subreflector and test it on the ground using long range

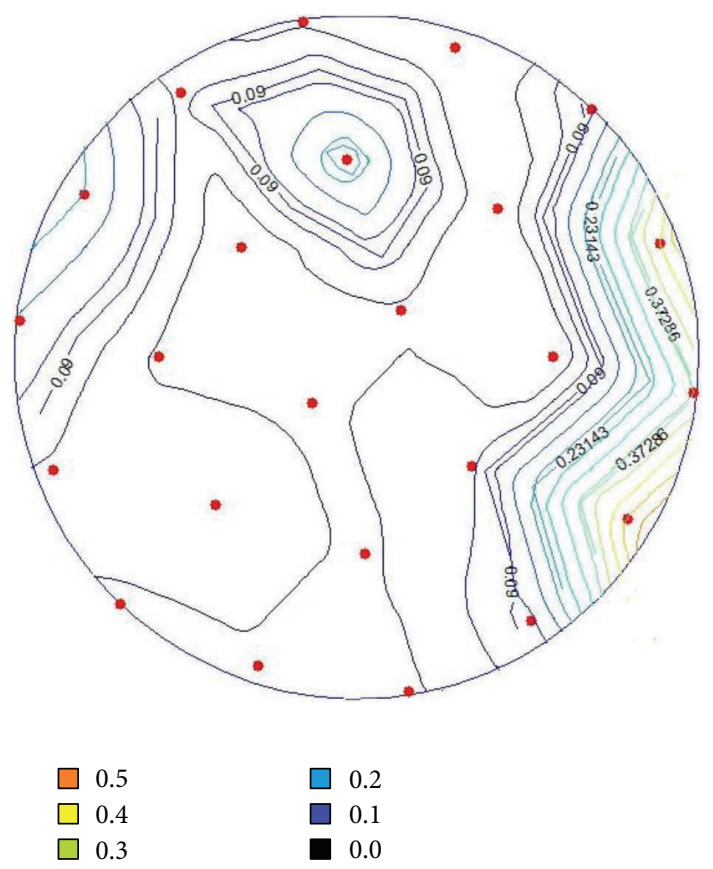

FIGURE 10: The changes in the subreflector's surface, presented as a topographic map. The lines are in $0.033 \mathrm{~cm}$ scale and represent the changes in height from the original (fixed) subreflector.

communication to LEO and GEO satellites. By using such model we should be able to further study the capabilities of beam-shaping and have a better understanding of the antenna pattern of such flexible-robotic antennas.

\section{Conflict of Interests}

The authors declare that there is no conflict of interests regarding the publication of this paper.

\section{Acknowledgment}

This research was partly supported by NSLComm. NSLComm (nslcomm.com) provides satellite manufacturers with unprecedented expandable antennas for satellite telecommunications.

\section{References}

[1] W. A. Imbriale, S. Gao, and B. Luigi, Space Antenna Handbook, Wiley Online Library, 2012.

[2] W. V. T. Rusch and P. D. Potter, Analysis of Reector Antennas, Academic Press, 2013.

[3] S. Rao, L. Shafai, and S. K. Sharma, Handbook of Reector Antennas and Feed Systems Volume III: Applications of Reectors, Artech House, 2013.

[4] R. L. Haupt, "Thinned arrays using genetic algorithms," IEEE Transactions on Antennas and Propagation, vol. 42, no. 7, pp. 993-999, 1994

[5] G. S. Hornby, A. I. Globus, D. S. Linden, and J. D. Lohn, "Automated antenna design with evolutionary algorithms," in Proceedings of the AIAA Space, AIAA 2006-7242, pp. 19-21, San Jose, Calif, USA, September 2006. 
[6] Y. T. Lo and S. W. Lee, "A study of space-tapered arrays," IEEE Transactions on Antennas and Propagation, vol. 14, no. 1, pp. 2230, 1966.

[7] O. Medina, A. Shapiro, and N. Shvalb, "Kinematics for an actuated flexible n-manifold," Journal of Mechanisms and Robotics, 2015.

[8] H. Yamada, S. Takaoka, and S. Hirose, "A snake-like robot for real-world inspection applications (the design and control of a practical active cord mechanism)," Advanced Robotics, vol. 27, no. 1, pp. 47-60, 2013.

[9] S. Kim, C. Laschi, and B. Trimmer, "Soft robotics: a bioinspired evolution in robotics," Trends in Biotechnology, vol. 31, no. 5, pp. 287-294, 2013.

[10] C. D. Onal, X. Chen, G. M. Whitesides, and D. Rus, "Sof mobile robots with on-board chemical pressure generation," in Proceedings of the International Symposium of Robotics Research (ISRR '11), pp. 1-16, Flagstaff, Ariz, USA, August-September 2011.

[11] S. Kim, E. Hawkes, K. Choy, M. Joldaz, J. Foleyz, and R. Wood, "Micro artificial muscle fiber using NiTi spring for sof robotics," in Proceedings of the IEEE/RSJ International Conference on Intelligent Robots and Systems (IROS '09), pp. 2228-2234, IEEE, St. Louis, Mo, USA, October 2009.

[12] L. Vink, V. Kan, K. Nakagaki et al., "Transform as adaptive and dynamic furniture," in Proceedings of the 33rd Annual ACM Conference Extended Abstracts on Human Factors in Computing Systems (CHI EA '15), pp. 183-183, ACM, Seoul, Republic of Korea, April 2015.

[13] M. De Berg, M. Van Kreveld, M. Overmars, and O. C. Schwarzkopf, Computational Geometry, Springer, 2000.

[14] TICRA RF Simulator Ltd, 2015, http://www.ticra.com/.

[15] MTI Wireless Edge Ltd, 2015, http://www.mtiwe.com/. 

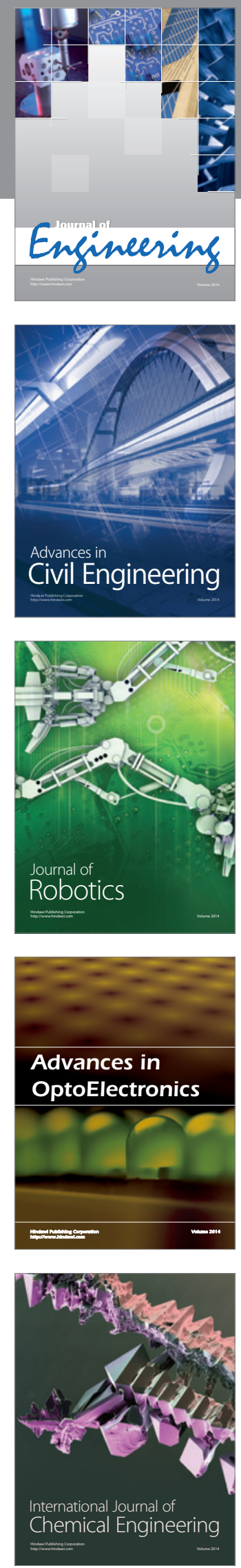

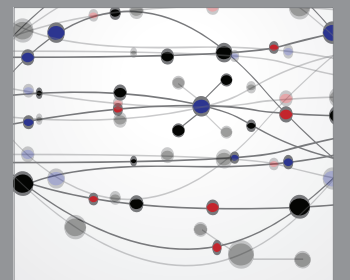

The Scientific World Journal
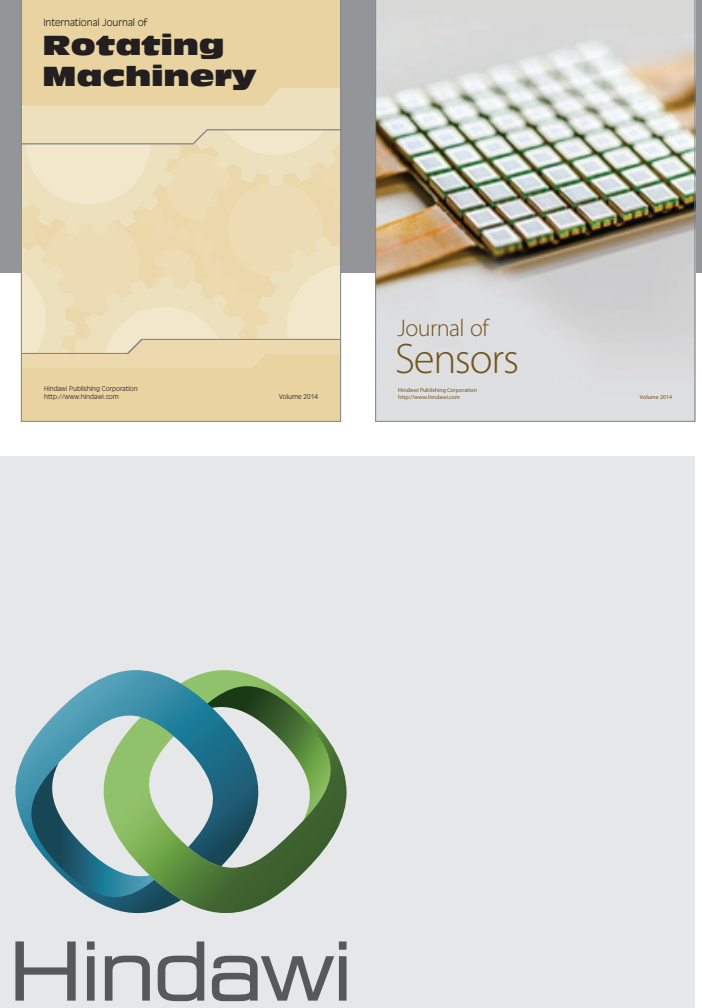

Submit your manuscripts at http://www.hindawi.com
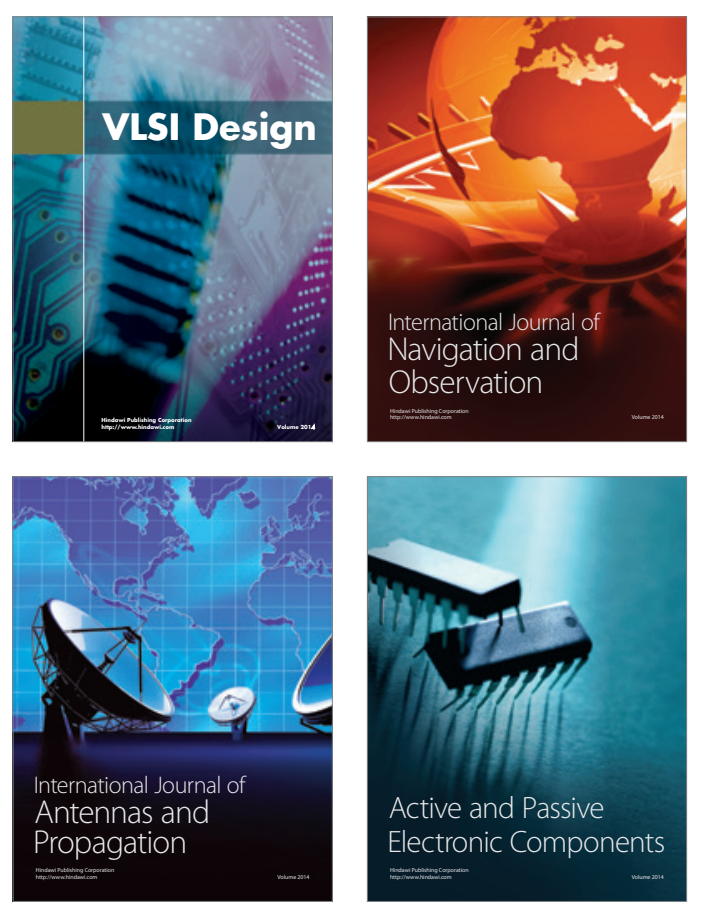
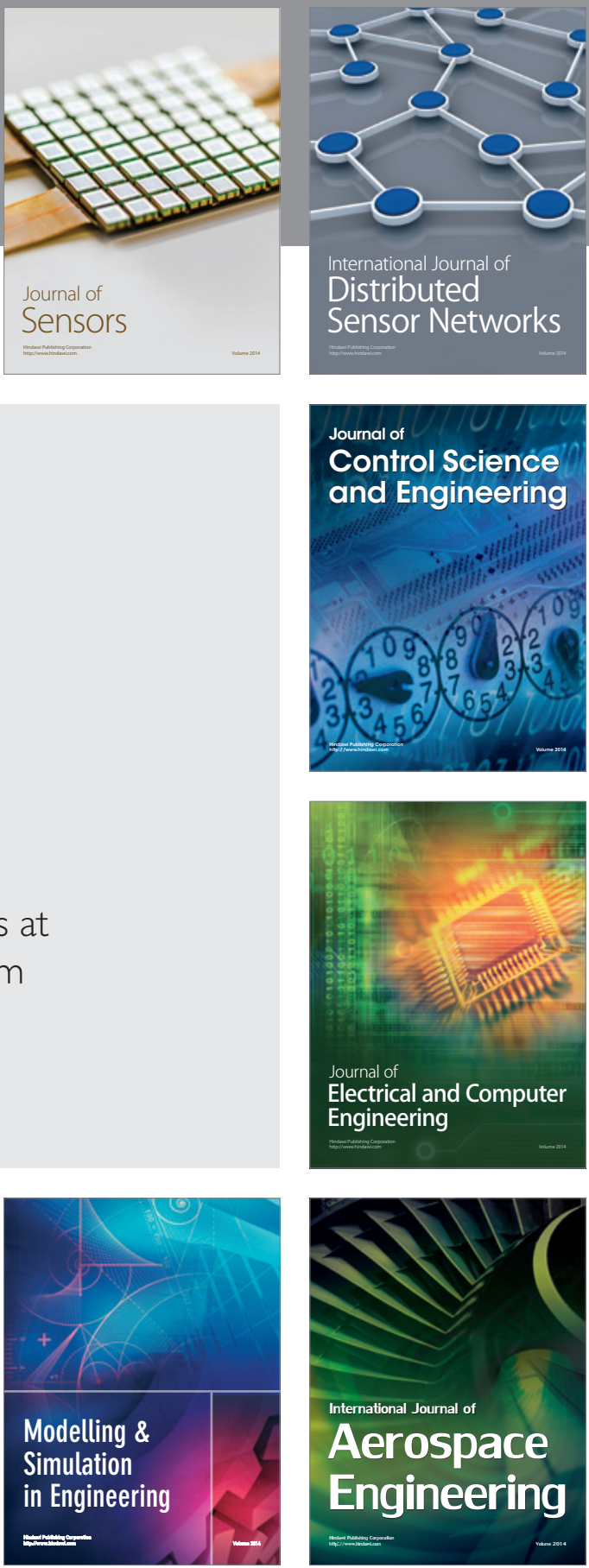

Journal of

Control Science

and Engineering
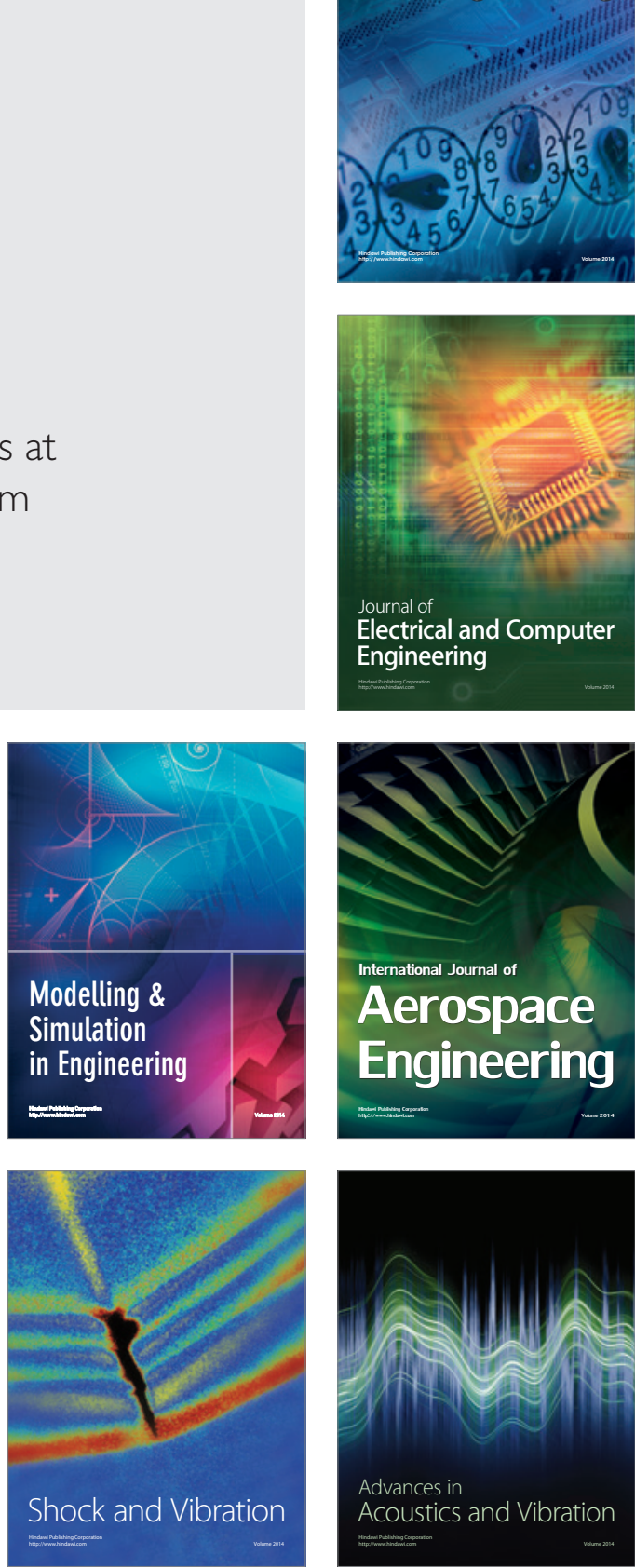